\title{
Auricular Acupuncture May Suppress Epileptic Seizures via Activating the Parasympathetic Nervous System: A Hypothesis Based on Innovative Methods
}

\author{
Wei He, ${ }^{1}$ Pei-Jing Rong, ${ }^{1}$ Liang Li, ${ }^{1}$ Hui Ben, ${ }^{1}$ Bing Zhu, ${ }^{1}$ and Gerhard Litscher ${ }^{1,2}$ \\ ${ }^{1}$ Institute of Acupuncture and Moxibustion, China Academy of Chinese Medical Sciences, Beijing 100700, China \\ ${ }^{2}$ Stronach Research Unit for Complementary and Integrative Laser Medicine, TCM Research Center Graz, \\ and Research Unit of Biomedical Engineering in Anesthesia and Intensive Care Medicine, Medical University of Graz, \\ Auenbruggerplatz 29, 8036 Graz, Austria \\ Correspondence should be addressed to Bing Zhu, zhubing@mail.cintcm.ac.cn \\ and Gerhard Litscher, gerhard.litscher@medunigraz.at
}

Received 12 October 2011; Accepted 26 October 2011

Academic Editor: Lu Wang

Copyright $\odot 2012$ Wei He et al. This is an open access article distributed under the Creative Commons Attribution License, which permits unrestricted use, distribution, and reproduction in any medium, provided the original work is properly cited.

\begin{abstract}
Auricular acupuncture is a diagnostic and treatment system based on normalizing the body's dysfunction. An increasing number of studies have demonstrated that auricular acupuncture has a significant effect on inducing parasympathetic tone. Epilepsy is a neurological disorder consisting of recurrent seizures resulting from excessive, uncontrolled electrical activity in the brain. Autonomic imbalance demonstrating an increased sympathetic activity and a reduced parasympathetic activation is involved in the development and progress of epileptic seizures. Activation of the parasympathetic nervous system such as vagus nerve stimulation has been used for the treatment of intractable epilepsy. Here, we propose that auricular acupuncture may suppress epileptic seizures via activating the parasympathetic nervous system.
\end{abstract}

\section{Introduction}

Epilepsy is a neurological disorder consisting of recurrent seizures resulting from excessive, uncontrolled electrical activity in the brain. Despite active pharmacological and neurosurgical treatments used for the treatment of epileptic disorders, the management of medically intractable epilepsy remains a difficult problem.

Over the past two decades, concerns regarding the side effects of pharmacological and neurosurgical approaches have increased interest in the use of complementary and alternative medicine (CAM) [1-3].

Autonomic imbalance is involved in the development and progress of epileptic seizures. Auricular acupuncture can treat diseases by increasing parasympathetic tone. Here, we propose that auricular acupuncture may suppress epileptic seizures via activating the parasympathetic nervous system.

\section{Auricular Acupuncture Can Increase Parasympathetic Tone}

Auricular acupuncture is a diagnostic and treatment system based on normalizing the body's dysfunction which is suggested to stimulate the peripheral reflexes, then activate these central brain pathways, and thus inhibit the maladaptive reflexes that contribute to neuropsychic disorders [4]. Auricular acupuncture was utilized to treat postoperative pain [5], improve neurorehabilitation [6], insomnia [7], and obesity [8] via modifying endorphinergic systems and the autonomic nervous system (ANS).

An increasing number of studies have demonstrated that auricular acupuncture has a significant effect on inducing parasympathetic tone. Manual ear acupressure at "heart" auricular acupoint induced a significant decrease in heart rate and a significant increase in heart rate variability total $[9$, 10]. Acupuncture on auricular acupoint "Shenmen" might 
calm the mind, slow down the heart rate, activate the parasympathetic nerves, and inhibit the sympathetic nerves [11]. Acupuncture conducted on the concha of the ear induces an increase in vagal activity [12]. During needling vision-related acupoints of ear acupuncture, mean blood flow velocity of the ophthalmic artery was significantly increased which may be induced by parasympathetic tone [13]. Another clinical study showed that stimulation of the ear induced a significant increase in the parasympathetic activity during the stimulation period of $25 \mathrm{~min}$ and during the poststimulation period of $60 \mathrm{~min}$ [14]. The external ear is innervated by several nerves, including vagus nerve, glossopharyngeal nerve, trigeminal nerve, facial nerve, and branches (the second and third) of the cervical spinal nerves [15]. The auricular branch of vagus nerve (ABVN) innervates the auricular concha and the external auditory meatus. Parasympathetic tone such as Arnold's reflexes has been clinically observed after stimulating innervation regions of the $\operatorname{ABVN}[16,17]$, which is considered as a bridge between the external ear and the internal organs [18]. In Traditional Chinese Medicine, auricular acupoints related to internal organs are located at the auricular concha [4]. Except for the ABVN, the glossopharyngeal nerve, the trigeminal nerve, and the facial nerve, all carry parasympathetic nerve fibers. Most nerves innervating the external ear carry parasympathetic components.

\section{Epilepsy Is Associated with Decreased Parasympathetic Tone}

Autonomic symptoms accompany all generalized tonicclonic seizures (GTC) and one-third of simple partial seizures. The ANS centers can be involved in complex partial, absence, and generalized tonic seizures. Measurements of ANS functions may be helpful in differentiating between epileptic seizures and nonepileptic psychogenic seizures [19]. The autonomic imbalance of epileptic seizures probably results from the hypersynchronized electrical impulse from the temporal and frontal areas to the limbic system, then to autonomic central nuclei in medulla including the nucleus tractus solitarius (NTS) and ambiguus nuclei. Both sympathetic and parasympathetic efferent discharges are then generated.

There is ample experimental and clinical proof that epilepsy goes along with autonomic imbalance demonstrating an increased sympathetic activity and a reduced parasympathetic activation. Novak et al. documented rapid parasympathetic withdrawal approximately 30 seconds before seizure onset and a sympathetic activation peak at seizure onset [20]. Temporal lobe epilepsy is known to be associated with ictal and interictal autonomic dysregulation, predominantly with sympathetic overactivity [21]. Higher sympathetic function and lower parasympathetic function have been demonstrated to be significant risk factors for sudden unexplained death in epilepsy subjects [22, 23].

Activation of the parasympathetic nervous system (PNS) has shown therapeutic benefits in brain diseases. Examples include vagus nerve stimulation (VNS) for epilepsy. VNS has been successfully applied for more than 20 years to treat drug-resistant epilepsy [24]. The antiseizure effect of VNS is considered to be mediated via vagal afferent projections to the NTS, then from the NTS to different brain regions which correlate with the pathogenesis of epilepsy [25]. Recently, VNS has also been applied for treatment of drug-resistant depression [26] and was suggested as a new approach for the treatment of heart failure [27] and stroke [28] by increasing the parasympathetic tone.

\section{Hypothesis}

Auricular acupuncture appears to modify the autonomic dysfunction by increasing parasympathetic activity. Thus, we hypothesize that auricular acupuncture may suppress epilepsy by increasing parasympathetic tone. We have done clinical trials and animal experiments on the effect and mechanism of auricular electroacupuncture for the treatment of epilepsy. In clinical trials, auricular electroacupuncture reduced seizure frequency and attenuated seizure severity. Animal results showed that auricular electroacupuncture suppressed epileptic discharges in electroencephalogram traces. All the results support our hypothesis.

\section{The Mechanism of Auricular Acupuncture for Epilepsy}

Acupuncture has been used to treat epilepsy. Acupoints selected to treat epilepsy included "GV 14" $[29,30]$, "ST 36" [31], and auricular acupoints such as "Pizhixia, Nao, and Shenmen" $[32,33]$. Most nerves innervating the external ear carry parasympathetic nerve fibers. Moreover, the ABVN is the only peripheral branch of the vagus nerve. Acupuncture at auricular acupoints especially in the area of auricular concha may induce vagal tone to suppress epileptic seizures. As the main vagal afferent, the NTS is considered as a neuroanatomical center for pathways of the antiseizure effect of auricular acupuncture [34]. Amelioration of illness by auricular acupuncture is believed to be through the reticular formation which is found to be histopathologically connected with focal-cortical seizure-induced generalized convulsive status epilepticus [35]. Recent findings highlight the possibility of inflammation in seizures and epileptogenesis [36]. Prototypical inflammatory cytokines such as IL- $1 \beta$, TNF- $\alpha$, and IL-6 have been shown to be overexpressed prominently by glia. Cytokines receptors are also upregulated, and the related intracellular signalling is activated in brain areas of seizure generation and propagation in experimental models of seizures [37]. The anti-inflammation effect perhaps is the mechanism of auricular acupuncture for epilepsy [38]. Possible mechanism of auricular acupuncture for the treatment of epilepsy is shown in Figure 1.

\section{Potential Application of Auricular Acupuncture for Other Diseases}

The ANS is the primary neural mediator of physiological responses to internal and external stimuli [39]. Functions of many or perhaps all visceral organs can be modulated by somatosympathetic or somatoparasympathetic reflex activity induced by an appropriate somatic afferent stimulation [40]. 


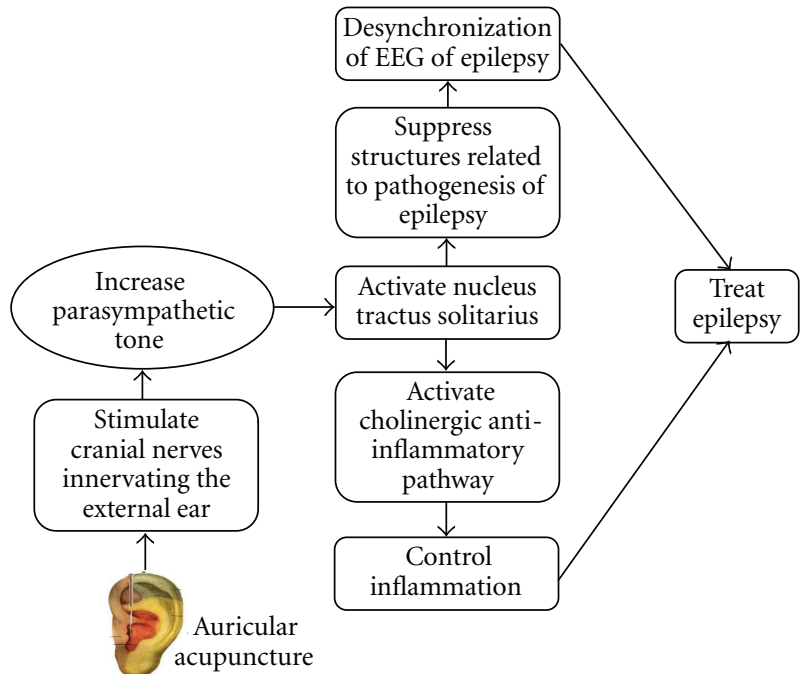

Figure 1: Possible mechanism of auricular acupuncture for the treatment of epilepsy.

According to the theory of Traditional Chinese Medicine, acupuncture has the function of bidirection-regulative effect. In addition to the dysfunction of parasympathetic system, auricular acupuncture can also modulate the dysfunction of sympathetic system [41]. Therefore, auricular acupuncture provides a somatic stimulation to treat diseases being induced or accompanied by an imbalance of the autonomic system.

VNS has been proposed to have the potential for the treatment of neuropsychiatric illnesses [42]. Yet it is an invasive procedure that may have potential side effects and complications. Its application in developing countries is limited for high costs. We can use auricular electroacupuncture, which is less invasive, of less cost, and convenient to treat diseases by setting up suitable parameters. Recently, kinds of acupuncture treatment instruments such as radio electric stimulator device [43] and P-Stim auricular electroacupuncture stimulation device [44] have been developed for the treatment of stress-related disorders and pain relief. Auricular vagal nerve stimulator is expected to be explored.

\section{Abbreviations}

CAM: Complementary and alternative medicine

GTC: Generalized tonic-clonic seizures

ANS: Autonomic nervous system

NTS: Nucleus tractus solitarius

VNS: Vagus nerve stimulation

PNS: Parasympathetic nervous system

ABVN: Auricular branch of vagus nerve.

\section{Acknowledgments}

This study was supported by the National Basic Research Program of China (973 program, no. 2011CB505201), the National Natural Science Foundation of China (no. 30901931), and the Beijing Natural Science Foundation (no. 7102120). The authors would like to thank Ingrid Gaischek,
MSc, for the help in paper preparation, and the German Society of Acupuncture (DAA) for support. Prof. Gerhard Litscher is also visiting Professor at the Institute of Acupuncture \& Moxibustion at the China Academy of Chinese Medical Sciences, Beijing, China.

\section{References}

[1] C.-H. Liu, Y.-W. Lin, N.-Y. Tang, H.-J. Liu, and C.-L. Hsieh, "Neuroprotective effect of Uncaria rhynchophylla in Kainic acid-induced epileptic seizures by modulating hippocampal mossy fiber sprouting, neuron survival, astrocyte proliferation, and S100b expression," Evidence-Based Complementary and Alternative Medicine, vol. 2012, Article ID 194790, 11 pages, 2012.

[2] T. N. Sathyaprabha, P. Satishchandra, C. Pradhan et al., "Modulation of cardiac autonomic balance with adjuvant yoga therapy in patients with refractory epilepsy," Epilepsy and Behavior, vol. 12, no. 2, pp. 245-252, 2008.

[3] A. W. Yuen and J. W. Sander, "Can slow breathing exercises improve seizure control in people with refractory epilepsy? A hypothesis," Epilepsy and Behavior, vol. 18, no. 4, pp. 331-334, 2010.

[4] L. Gori and F. Firenzuoli, "Ear acupuncture in European traditional medicine," Evidence-Based Complementary and Alternative Medicine, vol. 4, no. 1, pp. 13-16, 2007.

[5] G. N. Asher, D. E. Jonas, R. R. Coeytaux et al., "Auriculotherapy for pain management: a systematic review and meta-analysis of randomized controlled trials," Journal of Alternative and Complementary Medicine, vol. 16, no. 10, pp. 1097-1108, 2010.

[6] T. Oleson, "Auriculotherapy stimulation for neuro-rehabilitation," NeuroRehabilitation, vol. 17, no. 1, pp. 49-62, 2002.

[7] M. S. Lee, B. C. Shin, L. K. Suen, T. Y. Park, and E. Ernst, "Auricular acupuncture for insomnia: a systematic review," International Journal of Clinical Practice, vol. 62, no. 11, pp. 17441752, 2008.

[8] J. M. Lacey, A. M. Tershakovec, and G. D. Foster, "Acupuncture for the treatment of obesity: a review of the evidence," International Journal of Obesity, vol. 27, no. 4, pp. 419-427, 2003. 
[9] X. Y. Gao, S. P. Zhang, B. Zhu, and H. Q. Zhang, "Investigation of specificity of auricular acupuncture points in regulation of autonomic function in anesthetized rats," Autonomic Neuroscience, vol. 138, no. 1-2, pp. 50-56, 2008.

[10] X. Y. Gao, L. Wang, I. Gaischek, Y. Michenthaler, B. Zhu, and G. Litscher, "Brain-modulated effects of auricular acupressure on the regulation of autonomic function in healthy volunteers," Evidence-Based Complementary and Alternative Medicine, vol. 2012, Article ID 714391, 8 pages, 2012.

[11] C. C. Hsu, C. S. Weng, M. F. Sun, L. Y. Shyu, W. C. Hu, and Y. $\mathrm{H}$. Chang, "Evaluation of scalp and auricular acupuncture on EEG, HRV, and PRV," American Journal of Chinese Medicine, vol. 35, no. 2, pp. 219-230, 2007.

[12] R. La Marca, M. Nedeljkovic, L. Yuan, A. Maercker, and U. Ehlert, "Effects of auricular electrical stimulation on vagal activity in healthy men: evidence from a three-armed randomized trial," Clinical Science, vol. 118, no. 8, pp. 537-546, 2010.

[13] G. Litscher, "Computer-based quantification of traditional Chinese-, ear- and Korean hand acupuncture: needle-induced changes of regional cerebral blood flow velocity," Neurological Research, vol. 24, no. 4, pp. 377-380, 2002.

[14] E. Haker, H. Egekvist, and P. Bjerring, "Effect of sensory stimulation (acupuncture) on sympathetic and parasympathetic activities in healthy subjects," Journal of the Autonomic Nervous System, vol. 79, no. 1, pp. 52-59, 2000.

[15] N. Ueno, H. Sudo, Y. Hattori, K. Yuge, T. Miyaki, and H. Ito, "Innervation of the external ear in humans and the musk shrew," Nippon Jibiinkoka Gakkai Kaiho, vol. 96, no. 2, pp. 212-218, 1993.

[16] I. Tekdemir, A. Asian, and A. Elhan, "A clinico-anatomic study of the auricular branch of the vagus nerve and Arnold's earcough reflex," Surgical and Radiologic Anatomy, vol. 20, no. 4, pp. 253-257, 1998.

[17] D. Gupta, S. Verma, and S. K. Vishwakarma, "Anatomic basis of Arnold's ear-cough reflex," Surgical and Radiologic Anatomy, vol. 8, no. 4, pp. 217-220, 1986.

[18] D. Engel, "The gastroauricular phenomenon and related vagus reflexes," Archiv fur Psychiatrie und Nervenkrankheiten, vol. 227, no. 3, pp. 271-277, 1979.

[19] B. Müngen, M. S. Berilgen, and A. Arikanoğlu, "Autonomic nervous system functions in interictal and postictal periods of nonepileptic psychogenic seizures and its comparison with epileptic seizures," Seizure, vol. 19, no. 5, pp. 269-273, 2010.

[20] V. V. Novak, A. L. Reeves, P. Novak, P. A. Low, and F. W. Sharbrough, "Time-frequency mapping of R-R interval during complex partial seizures of temporal lobe origin," Journal of the Autonomic Nervous System, vol. 77, no. 2-3, pp. 195-202, 1999.

[21] M. D. Kanner and M. Andres, "Epilepsy and activity of the autonomic nervous system," Epilepsy Currents, vol. 2, no. 5, pp. 159-160, 2002.

[22] G. Rauscher, A. C. DeGiorgio, P. R. Miller, and C. M. DeGiorgio, "Sudden unexpected death in epilepsy associated with progressive deterioration in heart rate variability," Epilepsy and Behavior, vol. 21, no. 1, pp. 103-105, 2011.

[23] S. Mukherjee, M. Tripathi, P. S. Chandra et al., "Cardiovascular autonomic functions in well-controlled and intractable partial epilepsies," Epilepsy Research, vol. 85, no. 2-3, pp. 261-269, 2009.

[24] R. E. Elliott, A. Morsi, O. Tanweer et al., "Efficacy of vagus nerve stimulation over time: review of 65 consecutive patients with treatment-resistant epilepsy treated with VNS > 10years," Epilepsy and Behavior, vol. 20, no. 3, pp. 478-483, 2011.
[25] T. R. Henry and R. Thomas, "Therapeutic mechanisms of vagus nerve stimulation," Neurology, vol. 59, no. 6, supplement 4, pp. S3-S14, 2002.

[26] H. A. Sackeim, A. J. Rush, M. S. George et al., "Vagus nerve stimulation (VNS) for treatment-resistant depression: efficacy, side effects, and predictors of outcome," Neuropsychopharmacology, vol. 25, no. 5, pp. 713-728, 2001.

[27] H. U. Klein and G. M. Ferrari, "Vagus nerve stimulation: a new approach to reduce heart failure," Cardiology Journal, vol. 17, no. 6, pp. 638-643, 2010.

[28] C. Cheyuo, A. Jacob, R. Wu, M. Zhou, G. F. Coppa, and P. Wang, "The parasympathetic nervous system in the quest for stroke therapeutics," Journal of Cerebral Blood Flow and Metabolism, vol. 31, no. 5, pp. 1187-1195, 2011.

[29] J. L. Zhang, S. P. Zhang, and H. Q. Zhang, "Antiepileptic effects of electroacupuncture vs vagus nerve stimulation on cortical epileptiform activities," Journal of the Neurological Sciences, vol. 270, no. 1-2, pp. 114-121, 2008.

[30] J. L. Zhang, S. P. Zhang, and H. Q. Zhang, "Antiepileptic effect of electroacupuncture vs. vagus nerve stimulation in the rat thalamus," Neuroscience Letters, vol. 441, no. 2, pp. 183-187, 2008.

[31] J. Guo, J. Liu, W. Fu et al., "The effect of electroacupuncture on spontaneous recurrent seizure and expression of GAD67 mRNA in dentate gyrus in a rat model of epilepsy," Brain Research, vol. 1188, no. 1, pp. 165-172, 2008.

[32] J. Shu, R. Y. Liu, and X. F. Huang, "The effects of ear-point stimulation on the contents of somatostatin and amino acid neurotransmitters in brain of rat with experimental seizure," Acupuncture and Electro-Therapeutics Research, vol. 29, no. 12, pp. 43-51, 2004.

[33] J. Shu, R. Y. Liu, and X. F. Huang, "Efficacy of ear-point stimulation on experimentally induced seizure," Acupuncture and Electro-Therapeutics Research, vol. 30, no. 1-2, pp. 43-52, 2005.

[34] Y. O. Cakmak, "Epilepsy, electroacupuncture and the nucleus of the solitary tract," Acupuncture in Medicine, vol. 24, no. 4, pp. 164-168, 2006.

[35] N. Soliman and B. L. Frank, "Auricular acupuncture and auricular medicine," Physical Medicine and Rehabilitation Clinics of North America, vol. 10, no. 3, pp. 547-554, 1999.

[36] T. Granata, H. Cross, W. Theodore, and G. Avanzini, "Immune-mediated epilepsies," Epilepsia, vol. 52, supplement 3, pp. 5-11, 2011.

[37] A. Vezzani, S. Balosso, and T. Ravizza, "The role of cytokines in the pathophysiology of epilepsy," Brain, Behavior, and Immunity, vol. 22, no. 6, pp. 797-803, 2008.

[38] W. Y. Chung, H. Q. Zhang, and S. P. Zhang, "Peripheral muscarinic receptors mediate the anti-inflammatory effects of auricular acupuncture," Chinese Medicine, vol. 6, article 3, 2011.

[39] K. L. Teff, "Visceral nerves: vagal and sympathetic innervation," Journal of Parenteral and Enteral Nutrition, vol. 32, no. 5, pp. 569-571, 2008.

[40] A. Sato and R. F. Schmidt, "The modulation of visceral functions by somatic afferent activity," Japanese Journal of Physiology, vol. 37, no. 1, pp. 1-17, 1987.

[41] E. Y. Shen, C. L. Hsieh, Y. H. Chang, and J. G. Lin, "Observation of sympathomimetic effect of ear acupuncture stimulation for body weight reduction," American Journal of Chinese Medicine, vol. 37, no. 6, pp. 1023-1030, 2009. 
[42] D. A. Groves and V. J. Brown, "Vagal nerve stimulation: a review of its applications and potential mechanisms that mediate its clinical effects," Neuroscience and Biobehavioral Reviews, vol. 29, no. 3, pp. 493-500, 2005.

[43] S. Rinaldi, V. Fontani, L. Aravagli, and P. Mannu, "Psychometric evaluation of a radio electric auricular treatment for stress related disorders: a double-blinded, placebo-controlled controlled pilot study," Health and Quality of Life Outcomes, vol. 8, article 31, 2010.

[44] S. M. Sator-Katzenschalger and A. Michalek-Sauberger, "PStim auricular electroacupuncture stimulation device for pain relief," Expert Review of Medical Devices, vol. 4, no. 1, pp. 2332, 2007. 


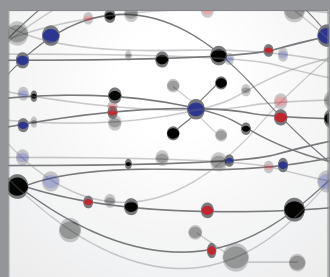

The Scientific World Journal
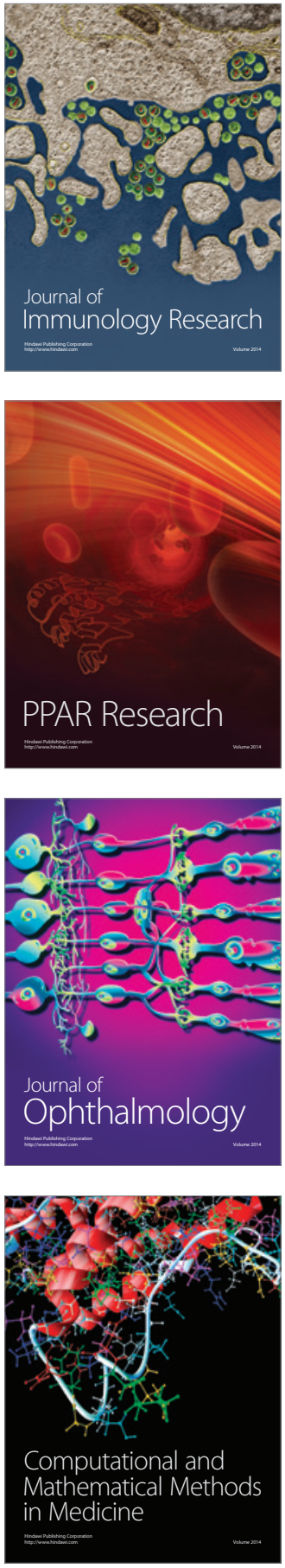

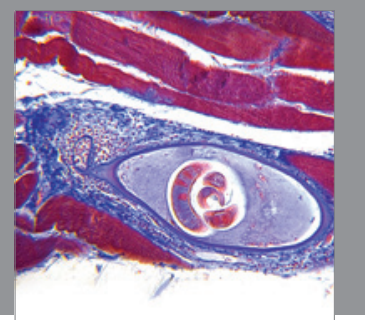

Gastroenterology

Research and Practice
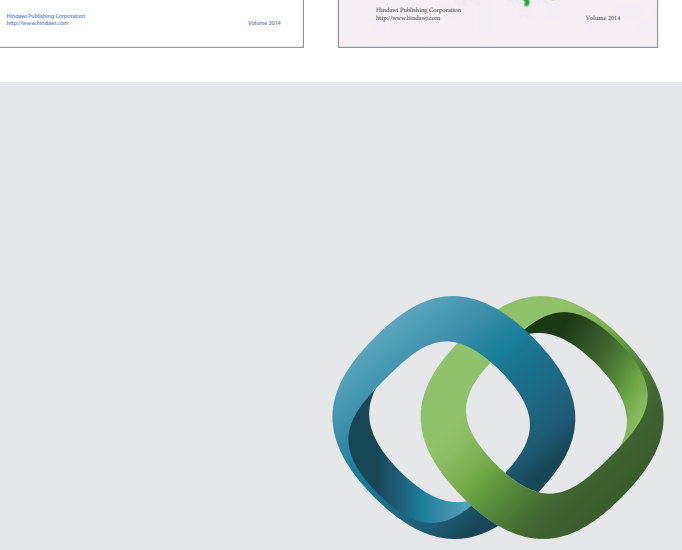

\section{Hindawi}

Submit your manuscripts at

http://www.hindawi.com
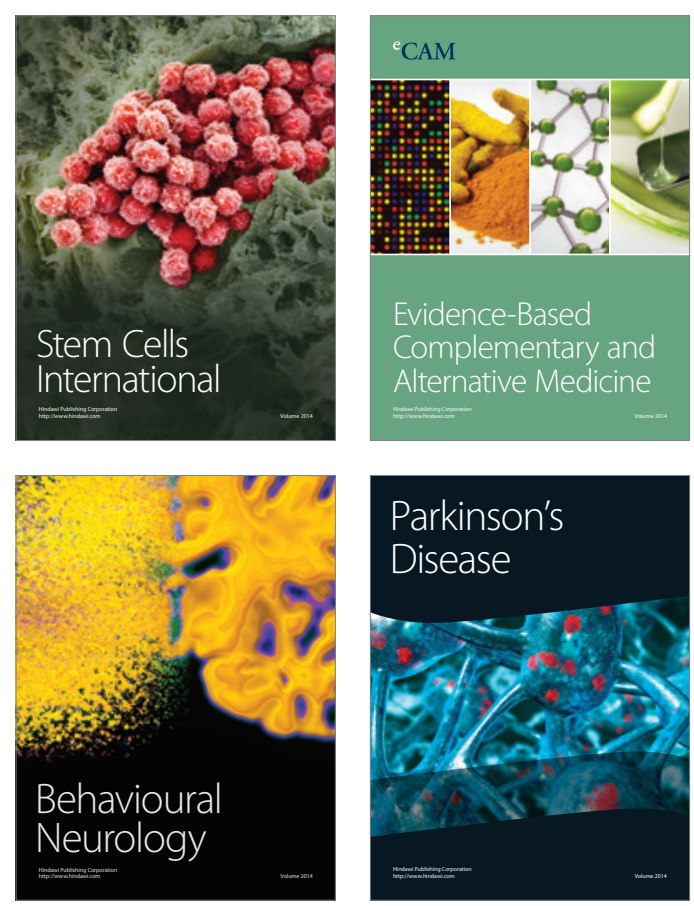

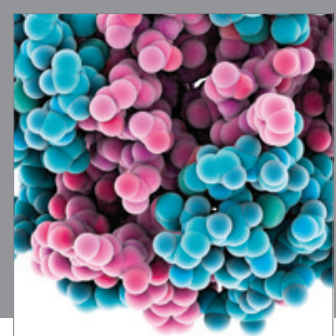

Journal of
Diabetes Research

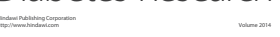

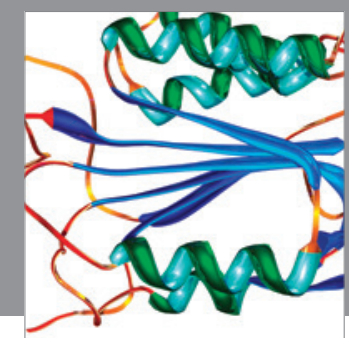

Disease Markers
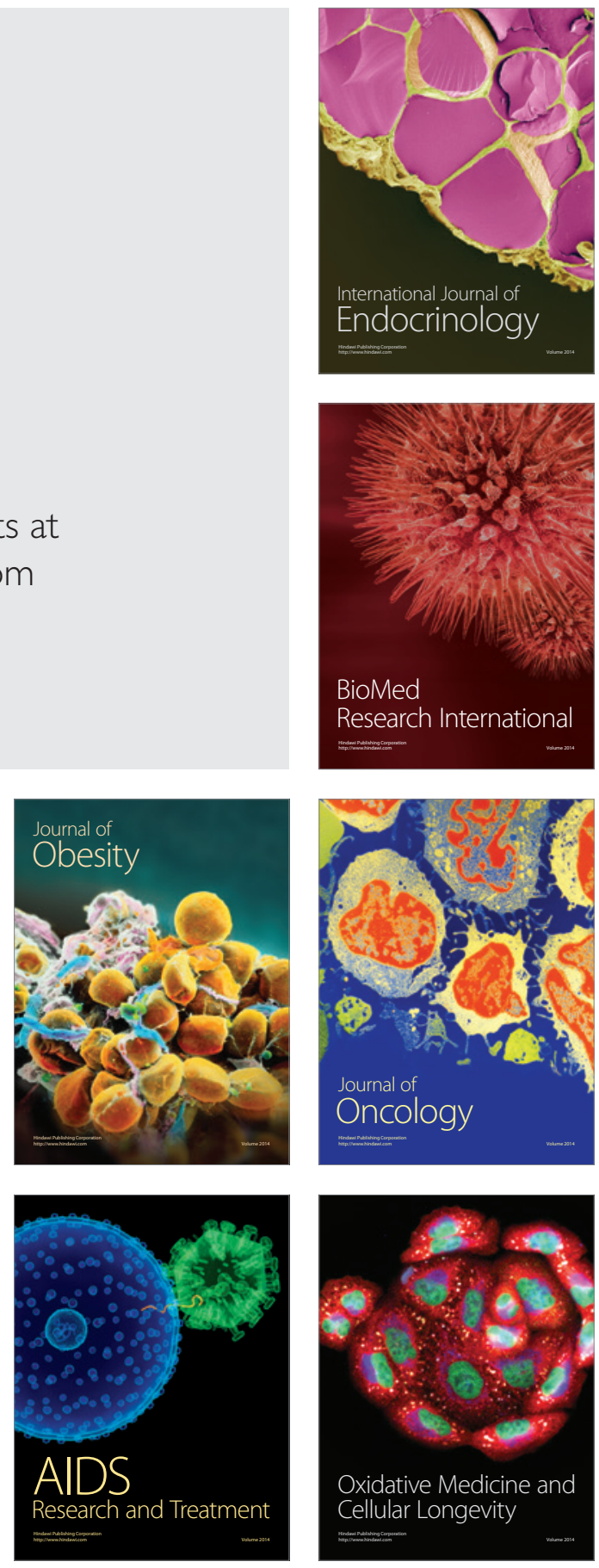